\title{
Perceived Racial Discrimination and Self- Esteem in African American Youth: Racial Socialization as a Protective Factor
}

\author{
April Harris-Britt, Cecelia R. Valrie, and Beth Kurtz-Costes \\ University of North Carolina
}

\author{
Stephanie J. Rowley \\ University of Michigan
}

\begin{abstract}
Racial socialization was examined as a protective factor that might buffer African American youth from the negative effects of perceived racial discrimination. Two types of racial socialization were examined: messages about race pride and preparation for bias. One hundred twenty-eight eighthgrade African American students participated in the study. As anticipated, both types of socialization moderated the relationship between discrimination and self-esteem. The negative relationship between perceived discrimination and self-esteem was mitigated for youth who reported more messages about race pride and a moderate amount of preparation for bias from their parents. In contrast, low race pride socialization and both high and low preparation for bias were associated with a negative relationship between perceived discrimination and self-esteem.
\end{abstract}

The rapid physical, psychological, and social changes that occur during adolescence have ramifications for individuals' evolving sense of identity and make youth vulnerable to a variety of risk factors (Caldwell, Kohn-Wood, Schmeelk-Cone, Chavous, \& Zimmerman, 2004). This period

Requests for reprints should be sent to Dr. April Harris-Britt, Department of Psychology, CB\# 3270 Davie Hall, University of North Carolina, Chapel Hill, NC 27599-3270. E-mail: ahb@unc.edu 
may be especially daunting for African American youth as they face the task of developing a positive sense of self as members of a minority group while becoming increasingly aware that society is stratified by ethnicity and race. Racial discrimination, defined as "beliefs, attitudes, institutional arrangements, and acts that tend to denigrate individuals or groups because of phenotypic characteristics or ethnic group affiliation," is often viewed as such a risk factor (Clark, Anderson, Clark, \& Williams, 1999, p. 805). Wong, Eccles, and Sameroff (2003) suggest that racism is seen as messages from the environment that individuals are not valued due to their race or ethnicity. Such messages may be particularly salient during adolescence, a vulnerable developmental period when the individual is formulating his or her identity and conceptualizations about the social world.

A large body of literature has linked perceived racial discrimination to deleterious physiological and psychological outcomes in African American adults (see Clark et al., 1999, for a review). More recently, researchers have begun to explore the effects of racial discrimination on child and adolescent populations. Results from a number of studies have linked experiences of discrimination and perceived racism to a range of psychosocial difficulties in African American youth, including low self-esteem and depressive symptoms (Caldwell et al., 2004; Clark, Coleman, \& Novak, 2004; DuBois, Burk-Braxton, Swenson, Tevendale, \& Hardesty, 2002; Fisher, Wallace, \& Fenton, 2000; Nyborg \& Curry, 2003; Prelow, Danoff-Burg, Swenson, \& Pulgiano, 2004; Wong et al., 2003). Nonetheless, relatively few studies have investigated possible protective factors that may buffer against the negative effects of perceived discrimination (see Caldwell et al., 2004; Sellers \& Shelton, 2003; Wong et al., 2003, for exceptions). Thus, the primary goals of the current study were to investigate the link between perceived racial discrimination and psychological well-being in African American adolescents and to examine perceived racial socialization messages as a moderator of the relationship between discrimination and self-esteem. In particular, we hypothesized that optimal racial socialization might buffer adolescents against the negative effects of discrimination.

\section{RACIAL SOCIALIZATION MESSAGES AS A BUFFER TO DISCRIMINATION}

Racial socialization-the implicit, explicit, purposeful, and unintended ways that parents' beliefs and behaviors convey views about race to 
children-has been cited as the primary practice utilized by African American families and communities to assist children in coping with race-related issues such as discrimination (Hughes, 2003). In the current project, we examine two types of socialization messages that African American parents transmit to their children: messages that instill racial pride and messages that prepare children for racism and bias. The majority of studies examining the influence of race pride socialization indicate that messages about cultural knowledge and pride are related to higher overall functioning and self-esteem (Caughy, O'Campo, Randolph, \& Nickerson, 2002; Constantine \& Blackmon, 2002; Hughes \& Chen, 1999; Stevenson, Reed, Bodison, \& Bishop, 1997; however, see Fisher et al., 2000, for null findings). In contrast, research examining the influence of preparation for bias messages on psychosocial outcomes has been more mixed. Some studies have found that parental preparation for bias is positively related to higher academic functioning, self-efficacy, and fewer depressive symptoms in adolescents (Bowman \& Howard, 1985; Hughes \& Chen, 1999; Stevenson et al., 1997). Other studies have found no relationship between preparation for bias and self-esteem or have linked preparation for bias to increased perceptions of discrimination and low academic functioning (Fisher et al., 2000; Hughes \& Chen, 1999). These studies have not examined possible interactions between discrimination and racial socialization in predicting adjustment.

To our knowledge, only one published study has examined racial socialization as a possible moderator between discrimination and psychological well-being. Fischer and Shaw's (1999) investigation with African American college students, ages 18-25, found that the negative relationship between perceived racism and overall mental health was significant at low levels (.5SD below mean) of bias preparation but nonsignificant at high levels (.5SD above mean) of bias preparation. These findings, along with the equivocal results from previous studies, might suggest a curvilinear relationship between bias preparation and outcomes, whereby some preparation for bias is necessary for healthy adjustment, yet an overemphasis on racial barriers may weaken the child's sense of self-efficacy and worth (as posited by Hughes \& Johnson, 2001). It is important to note that Fischer and Shaw (1999) only focused on reports of messages related to racism and barriers, without exploring the possible effects of cultural pride messages. Furthermore, racial socialization may be an even stronger moderator of the impact of discrimination among adolescents, as compared with young adults, given the greater daily proximity to their parents and developmental stage. 


\section{THE CURRENT STUDY}

In the current study, we hypothesized that adolescents' reports of racial socialization messages would moderate the relationship between perceived discrimination and self-esteem, and that the nature of the moderating effect would be determined by the type of socialization messages. Because racial pride messages focus on positive characteristics and significant contributions of one's racial group, such messages were expected to serve as a direct (linear) buffer against the effects of perceived discrimination. In contrast, preparation for bias messages were expected to serve as a buffer against perceptions of discrimination in a curvilinear fashion, such that either too little preparation for racial barriers or an overemphasis on discrimination and prejudice may exacerbate the negative relationship between discrimination and self-esteem. Thus, this study makes two important contributions to the research literature: racial socialization is examined as a protective factor in the relationship between perceived discrimination and self-esteem among young adolescents; and both linear and curvilinear effects of racial pride socialization and preparation for discrimination were tested.

\section{METHOD}

\section{Participants}

Participants were 128 eighth-grade African American students $(M$ age $=$ 13.9 years; $S D=.67 ; 68$ girls, 60 boys), recruited from the only two public middle schools located in a rural town in the southeastern region of the United States. According to 2000 U.S. Census Bureau data, African Americans comprise $48.3 \%$ of the county school district. School 1 had a student population that was about $70 \%$ African American with $61 \%$ of the students at the school eligible for free/reduced school lunch. School 2 had a population that was $66 \%$ African American with $75 \%$ of students eligible for free/reduced lunch. About half (51.5\%) of the students lived in married, two-parent households. Approximately 59\% of the participants lived in households that reported annual incomes below \$30,000, 21\% lived in households with incomes between $\$ 30,000$ and $\$ 50,000$, and $20 \%$ lived in households with incomes exceeding $\$ 50,000$ per year.

\section{Procedure}

Informed parental consent and child assent were obtained for all participants after forms were distributed to students in their classrooms. 
Approximately $80 \%$ of students returned forms, with the majority (95\%) agreeing to participate. Adolescents completed questionnaires in groups at their school as part of a larger study about stereotypes. Each student received a $\$ 5$ gift certificate for participation. Family demographic data were obtained when parents completed a questionnaire that was mailed to the home as part of the larger study.

\section{Measures}

Perceived discrimination. Adolescents were read the following prompt:

Sometimes people are treated badly because of a group to which they belong. For example, sometimes girls aren't allowed to play football with boys in their neighborhood just because they're girls. This is called "discrimination." We have some questions below that are related to race or ethnic discrimination-in other words, related to being Black, White, Hispanic, Asian, etc.

Perceptions of discrimination across institutional, educational, and peer contexts were then measured using the Adolescent Discrimination Distress Index (ADDI; Fisher et al., 2000), which consists of 15 items and asks students to rate on a 5 -point scale $(1=$ never, $2=$ once or twice, $3=$ 3-5 times, $4=6-10$ times, $5=$ more than 10 times) how often they have experienced each of the described events during the past 3 months. Representative items include, Because of your race or ethnicity... "You were given a lower grade than you deserved," and "You received poor service at a restaurant or store." Adolescents' responses to the items were averaged to yield a single score indicating the frequency of their discrimination experiences, with higher scores indicating greater levels of perceived discrimination. Fisher et al. (2000) reported strong test-retest reliability for the measure. The ADDI items assess discrimination in three contexts: peers, school, and institutions. Bivariate correlations between the three subscales ranged from .53 to .88 ( $p>.001)$ in our sample; thus, a composite average across items was utilized. Excellent internal consistency for the composite score was found in the present sample $(\alpha=.83)$.

Racial socialization. Adolescents' perceptions of racial socialization were assessed using the Racial Socialization Scale (Hughes \& Chen, 1997), which assesses two types of racial socialization messages, race pride (e.g., "Talked about being proud to be Black") and preparation for bias (e.g., "Said that people might treat you badly due to race"). Students rated each 
item on a 5 -point scale $(1=$ never, $2=$ once or twice, $3=3-5$ times, $4=6-$ 10 times, $5=$ more than 10 times), indicating how often their parents had engaged in the socialization behavior during the past year. Mean scores were calculated for the race pride (eight items; $\alpha=.74$ ) and preparation for bias (nine items; $\alpha=.84$ ) subscales.

Self-esteem. Adolescents completed the Rosenberg Self-Esteem Inventory (RSE; Rosenberg, 1965) to assess their general feelings of acceptance and global self-esteem. Each of the 10 items was rated on a 4-point scale ranging from 1 (strongly disagree) to 4 (strongly agree) (e.g., "I have a positive attitude toward myself"). Negative items were reverse coded (e.g., "I certainly feel useless at times") and the mean of all items was calculated, with higher scores reflecting more positive self-esteem. Test-retest reliability and validity have been established in prior research, and internal consistency was strong in the present sample $(\alpha=.76)$.

\section{DATA ANALYTIC STRATEGY}

Our primary hypotheses were tested using two separate stepwise multiple regression equations to examine the linear and curvilinear effects of each form of racial socialization (race pride; preparation for bias) on the relationship between perceived discrimination and self-esteem. In the first step of each regression equation, adolescents' age and gender, parents' marital status, parental education level, and household income were entered as control variables, along with perceptions of discrimination and the type of racial socialization. The second step of each equation included the two-way interaction of perceptions of discrimination with the type of racial socialization. In the final step, the quadratic term for the race socialization type (the square of the mean-centered value) and the product of perceived discrimination and the quadratic term for the race socialization type were added to the model to predict self-esteem. Finally, post hoc analyses were conducted to probe significant interactions.

\section{RESULTS}

The majority of the students (94\%) reported experiencing at least one discriminatory event within the past 3 months. The most frequently experienced events were people expecting more of (you) than others your age $(67 \%)$, receiving a lower grade than deserved $(66 \%)$, being wrongly disciplined or given after-school detention (63\%), and being 
called racially insulting names by other kids at school (50\%). The majority of students (94\%) indicated that their parents had conveyed messages of race pride, and all of the students reported exposure to preparation for bias messages.

Descriptive statistics and bivariate analyses among key study variables are shown in Table 1. None of the variables were significantly skewed, all $p^{\prime} \mathrm{s}>.50$. Analysis of variance (ANOVA) results revealed no significant mean differences between adolescents' perceptions of discrimination, parental racial socialization practices, or self-esteem as a function of adolescents' gender, school attended, parent education level, parental marital status, or household income.

\section{Race Pride, Perceived Discrimination, and Adolescent Self-Esteem}

Results of regression analyses examining the first hypothesis-that race pride would serve as a direct or linear buffer against the effects of perceived discrimination on self-esteem-are shown in Table 2. The first step was not significant, $F(7,85)=1.59, p<.15$, and the model only accounted for $4 \%$ of the variance in predicting adolescents' self-esteem. Contrary to expectations, the second step was also nonsignificant, $F(8,84)=1.59$, $p<.15$, and the two-way interaction between perceived discrimination and race pride did not contribute additional variance in predicting selfesteem, $\Delta F(1,84)=1.51, p=.22$. The overall model was significant at the final step, $F(10,82)=2.43, p<.05$ and accounted for $12 \%$ of the variance in predicting adolescents' self-esteem, $\Delta F(2,82)=4.29, p<.05$. The main effect for race pride and the interaction between perceived discrimination and race pride were both qualified by a significant interaction between

TABLE 1

Descriptive Statistics and Correlations for Study Variables

\begin{tabular}{lrccccr}
\hline & $M(S D)$ & 1 & 2 & 3 & 4 & 5 \\
\hline 1. Age (years) & $13.87(.63)$ & - & & & \\
2. Perceived discrimination & $1.83(.65)$ & .07 & - & & \\
3. Preparation for bias & $2.31(.88)$ & $-.18^{*}$ & $.27^{* * *}$ & - & & \\
4. Race pride & $2.52(.82)$ & -.12 & $.34^{* * * *}$ & $.68^{* * * *}$ & - & - \\
5. Self-esteem & $3.40(.47)$ & $-.28^{* * *}$ & -.08 & $.26^{* * *}$ & $.24^{* *}$ & - \\
\hline
\end{tabular}

Note. $N=128$.

$* p<.05 ; * * p<.01 ; * * * * x<.001$. 
TABLE 2

Regression Analyses Examining Curvilinear Effects of Racial Socialization Messages on the Relationship Between Perceived Discrimination and Self-Esteem

\begin{tabular}{|c|c|c|c|c|c|c|c|}
\hline & \multicolumn{3}{|c|}{ Step 1} & \multicolumn{2}{|c|}{ Step 2} & \multicolumn{2}{|c|}{ Step 3} \\
\hline & $B$ & $S E$ & $\beta$ & $B \quad S E$ & $\beta$ & $S E$ & $\beta$ \\
\hline \multicolumn{8}{|l|}{ Race pride models } \\
\hline Gender & .07 & .09 & .08 & .07 .09 & .08 & .04 .09 & .05 \\
\hline Age & -.01 & .01 & -.20 & -.01 .01 & -.20 & -.01 .01 & -.18 \\
\hline Parental marital status & -.04 & .03 & -.13 & -.03 .03 & -.12 & -.04 .03 & -.14 \\
\hline Parental income & .01 & .02 & .05 & .01 .02 & .08 & .02 .02 & .11 \\
\hline Parental education & -.03 & .03 & -.14 & -.03 .03 & -.15 & -.03 .02 & -.13 \\
\hline Discrimination & -.06 & .07 & -.10 & -.09 .07 & -.14 & .03 .08 & .05 \\
\hline Race pride & .10 & .05 & .20 & .11 .05 & $.22 *$ & .15 .06 & $.29 *$ \\
\hline Discrimination $\times$ Race pride & & & & .10 .08 & .13 & .23 .09 & $.30 *$ \\
\hline Race pride $^{2}$ & & & & & & -.01 .06 & -.02 \\
\hline Discrimination $\times$ Race pride ${ }^{2}$ & & & & & & .08 & $-.42 *$ \\
\hline \multicolumn{8}{|l|}{ Preparation for bias models } \\
\hline Gender & .06 & .09 & .07 & .05 .09 & .06 & .01 .09 & .01 \\
\hline Age & -.01 & .01 & -.19 & -.01 .01 & -.18 & -.01 .01 & -.19 \\
\hline Parental marital status & -.03 & .03 & -.12 & -.03 .03 & -.12 & -.06 .03 & -.22 \\
\hline Parental income & .01 & .02 & .05 & .01 .02 & .05 & -.00 .02 & -.01 \\
\hline Parental education & -.03 & .03 & -.15 & -.03 .03 & -.14 & -.03 .02 & -.14 \\
\hline Discrimination & -.06 & .07 & -.09 & -.06 .07 & -.10 & .11 .09 & .18 \\
\hline Preparation for bias & .11 & .05 & $.23 *$ & .11 .05 & $.23^{*}$ & .08 .06 & .17 \\
\hline Discrimination $\times$ Preparation for bias & & & & .07 .08 & .09 & .26 .10 & $.33 *$ \\
\hline Preparation for bias $^{2}$ & & & & & & .08 .06 & .20 \\
\hline Discrimination $\times$ Preparation for bias ${ }^{2}$ & & & & & & -.26 .09 & $-.51 *$ \\
\hline
\end{tabular}

Note. Gender $(0=$ girl, $1=$ boy $)$ and parental marital status $(0=$ not married, $1=$ married $)$. ${ }^{*} p<.05$.

perceived discrimination and the squared term for race pride, $t(92)=2.50$, 2.45 , and 2.91, respectively, all $p^{\prime} \mathbf{s}<.05$.

\section{Preparation for Bias, Perceived Discrimination, and Adolescent Self-Esteem}

The results of stepwise regression analyses examining the second hypothesis-that preparation for bias socialization would serve as a buffer against the effects of perceived discrimination in a curvilinear fashionare also shown in Table 2. Neither Step $1, F(7,85)=1.79, p<.10$, nor Step 2, 
TABLE 3

Post Hoc Tests of Linear-Curvilinear Interaction Between Perceived Discrimination and Racial Socialization on Self-Esteem

\begin{tabular}{lcccccccc}
\hline & \multicolumn{3}{c}{ Race Pride } & & \multicolumn{3}{c}{ Preparation for Bias } \\
\cline { 2 - 4 } \cline { 6 - 8 } Level of Race Socialization & Simple Slope & Variance & t-value & & Simple Slope & Variance & $t$-value \\
\hline$+2.0 S D$ & -.2042 & .0259 & -1.27 & & -.3626 & .0292 & $-2.12^{*}$ \\
$+1.5 S D$ & -.0288 & .0109 & -.28 & & -.1923 & .0129 & $-1.69^{*}$ \\
$+1.0 S D$ & -.0694 & .0071 & .82 & & .0770 & .0098 & .78 \\
$+.5 S D$ & .0902 & .0069 & 1.09 & & .1452 & .0093 & 1.51 \\
Mean & .0338 & .0069 & .41 & & .1124 & .0073 & 1.32 \\
$-.5 S D$ & .0902 & .0069 & 1.09 & & -.0213 & .0064 & -.27 \\
$-1.0 S D$ & -.3110 & .0155 & $-2.50^{*}$ & -.2561 & .0158 & $-2.04^{*}$ \\
$-1.5 S D$ & -.5994 & .0385 & $-3.05^{*}$ & -.5920 & .0513 & $-2.61^{*}$ \\
$-2.0 S D$ & -.9651 & .0907 & $-3.21^{*}$ & -1.029 & .1354 & $-2.80^{*}$ \\
\hline
\end{tabular}

Note. Simple slope and variance rounded for presentation in table but not during $t$-test calculations.

${ }^{*} p<.05(d f=90)$.

$F(8,84)=1.65, p<.15$, were significant, with both steps only accounting for $6 \%$ of the variance in predicting adolescents' self-esteem, $\Delta F(1,84)=.67, p=.41$. The overall model was significant at the final step, $F(10,82)=2.43, p<.05, \Delta F(2,82)=4.93, p<.05$, and accounted for $13 \%$ of the variance in predicting adolescents' self-esteem. The two-way interaction between perceived discrimination and preparation for bias was significant, $t(92)=2.50, p<.05$, but was qualified by the significant interaction between perceived discrimination and the squared term for preparation for bias, $t(92)=3.04, p<.01$.

\section{Probing the Interactions}

Aiken and West's (1991) procedures and guidelines were used to interpret the meaning of the significant linear-quadratic interactions. For each form of racial socialization, the regression equations were solved at high and low levels of the linear factor (discrimination) and conditional values ranging from $+2 S D$ above to $-2 S D$ below the mean of the quadratic terms (preparation for bias and race pride, separately). The simple slope $\left(b_{3}+b_{4} X+b_{5} X^{2}\right)$ and variance of the simple slope $\left(s_{33}+X^{2} s_{44}+2 X s_{34}+2 X^{2} s_{35}+2 X^{3} s_{45}\right)$ were then computed and used to calculate a $t$-test to determine if each line was statistically significant from zero.

As seen in Table 3, the negative relationship between perceived discrimination and self-esteem was significant when levels of race pride 
socialization were at $<-1 S D$ below the mean but was not significantly different from zero when race pride socialization was at or above the mean. In contrast, the negative relationship between perceived discrimination and self-esteem was significant when levels of preparation for bias were at $<-1 S D$ below the mean and at more than $+1.5 S D$ above the mean. However, the relationship between perceived discrimination and self-esteem was not significant when levels of preparation for bias were more normative (i.e., within the range of $-.5 S D$ below to +1 $S D$ above the mean). The negative relationship between self-esteem and perceptions of discrimination was more pronounced in the absence of preparation for bias as compared to in the presence of such socialization efforts.

\section{DISCUSSION}

This study adds to the growing literature focused on the effects of perceived racial discrimination on African American adolescents. The present results suggest that some of the negative effects associated with perceiving oneself to be the victim of racial discrimination may be mitigated if adolescents have been adequately socialized to deal with such experiences. These results may help to explain why there has not always been a consistent link between perceived discrimination and some indicators of social and emotional well-being (Fischer \& Shaw, 1999). Not until one examines the relative influence (i.e., the quantity or frequency) of racial socialization messages can the presence or absence of this relationship be adequately understood.

This study extends existing research by examining a unique interactive effect of perceived discrimination and racial socialization on self-esteem in a sample of African American adolescents. Qualitatively, adolescents' reports of race pride messages appeared to serve as a direct buffer on this relationship, as perceptions of discrimination were associated with lower self-esteem for adolescents who reported minimal exposure to positive messages about their heritage and culture, whereas adolescents who reported more frequent exposure to race pride messages did not seem to be negatively affected by perceived discrimination.

Consistent with our hypotheses, the buffering effects of preparation for bias do not seem to operate in the same manner as racial pride messages, because preparation for bias had a curvilinear influence on the relationship between perceptions of discrimination and self-esteem. At low levels of preparation for bias, greater perceptions of discrimination were associated with lower self-esteem. Given moderate levels of bias preparation, 
discrimination was not significantly predictive of self-esteem, suggesting that these messages might protect the young adolescent's ego and sense of self in the face of discrimination. Taken together, these results support theories that suggest that African American adolescents who do not understand racism and their unique cultural heritage may be handicapped in terms of their ability to cope effectively with racism (Stevenson et al., 1997).

Perhaps the most interesting result of the study is the finding that the negative relationship between perceived discrimination and self-esteem reappears at high levels of preparation for bias. This result supports prior theory suggesting that feelings of devaluation that may result from discriminatory experiences might be intensified by a frequent focus on racism (Hughes \& Johnson, 2001; Stevenson et al., 1997). Parental messages that signal to youth that they will consistently and persistently face prejudice and discrimination because of their race (a personal and internal factor), regardless of their own effort, may be maladaptive. Thus, an overemphasis on bias-that is, parents' frequent reminders of racial barriers-may lead adolescents to feel helpless and lacking control over their environment and consequently result in lower self-esteem.

\section{Suggestions for Future Research}

The sample for the current study was drawn from predominately African African middle schools in the Southeastern United States and thus is not representative of students attending schools and living in neighborhoods that are more socioeconomically and ethnically diverse. Discrimination might have an even stronger negative influence on psychosocial outcomes-and thus, racial socialization might be even more important as a buffer for African American students-in majority White schools. It will be important to test the application of these models with students in contexts in which they are in the minority, as well as environments that are more heterogeneous in racial makeup.

A second consideration for future research is that all data in the current study were self-report. We elected to use adolescent reports of parental racial socialization rather than parent reports because we believed that adolescents' perceptions of the frequency of race messages were more important than an objective measure of such socialization. Adolescent reports of discrimination were used for the same reason. Others have argued that one's perception of an experience, not merely the experience, influences normal developmental processes (e.g., Spencer, 1999). A risk of using adolescent reports for all measures is that they may not be veridical. In this case, adolescents' perceptions of discrimination and/or 
their self-esteem may have influenced their reports of parenting. Therefore, studies utilizing independent reports of both racial socialization and discrimination are needed to provide converging evidence for the buffering effects of parents' race-related messages and to better inform intervention efforts at the child and parent level.

Another important area for future research is to better understand how racial socialization occurs within African American families. Are most conversations about race prompted by discriminatory experiences? To what extent are such conversations elicited by the child versus initiated by the parent? Are youth more likely to remember conversations about discrimination than those related to racial pride? Our knowledge about racial socialization, as well as our understanding of youth's experiences of racial discrimination, will be enriched through the use of qualitative methods (cf. Rosenbloom \& Way, 2004).

Finally, the data used to test our hypotheses were gathered at a single time point. Consequently, causal relationships and temporal ordering cannot be established. Although we hypothesized that perceptions of discrimination and parental messages about race interact to influence adolescents' self-esteem, it is also likely that adolescents with low selfesteem are more likely to perceive their environment as more discriminatory than adolescents with high levels of self-esteem. Similarly, it is plausible that adolescents' views about their parents' race-related messages may be linked to increases in their awareness or perceptions of discrimination. These pathways are undoubtedly bidirectional by virtue of the transactional nature of parent-child interactions. Longitudinal studies examining various indices of adjustment will be integral to further clarify these temporal orders, to better understand age-related changes in racial socialization and perceptions of discrimination, and to elucidate moderating and mediating mechanisms and processes across childhood and adolescence.

\section{ACKNOWLEDGMENTS}

This manuscript is based upon work supported by the National Institutes of Health under Grant No. 5 R03 HD-44052-2 awarded to Beth Kurtz-Costes and Stephanie J. Rowley. The authors thank Ndidi Okeke, Dana Wood, and Taniesha Woods and members of the Youth Identity Project lab for assistance with data collection. We are grateful to participating students, families, and school personnel who made this project possible. 


\section{REFERENCES}

Aiken, L. S., \& West, S. G. (1991). Multiple regression: Testing and interpreting interactions. London, U.K.: Sage Publications.

Bowman, P. J., \& Howard, C. (1985). Race-related socialization, motivation, and academic achievement: A study of Black youths in three-generation families. Journal of the American Academy of Child Psychiatry, 24, 134-141.

Caldwell, C. H., Kohn-Wood, L. P., Schmeelk-Cone, K. H., Chavous, T. M., \& Zimmerman, M. A. (2004). Racial discrimination and racial identity as risk and protective factors for violent behaviors in African American young adults. American Journal of Community Psychology, 33, 91-105.

Caughy, M. O., O'Campo, P. J., Randolph, S. M., \& Nickerson, K. (2002). The influence of racial socialization practices on the cognitive and behavioral competence of African American preschoolers. Child Development, 73, 1611-1625.

Clark, R., Anderson, N. B., Clark, V. R., \& Williams, D. R. (1999). Racism as a stressor for African Americans: A biopsychosocial model. American Psychologist, 54, 805-816.

Clark, R., Coleman, A., \& Novak, J. (2004). Initial psychometric properties of the everyday discrimination scale in black adolescents. Journal of Adolescence, 27, 363-368.

Constantine, M., \& Blackmon, S. (2002). Black adolescents' racial socialization experiences: Their relations to home, school, and peer self-esteem. Journal of Black Studies, 32, 322-335.

DuBois, D. L., Burk-Braxton, C., Swenson, L. P., Tevendale, H. D., \& Hardesty, J. L. (2002). Race and gender influences on adjustment in early adolescence: Investigation of an integrative model. Child Development, 73, 1573-1592.

Fischer, A. R., \& Shaw, C. M. (1999). African Americans' mental health and perceptions of racist discrimination: The moderating effects of racial socialization experiences and selfesteem. Journal of Counseling Psychology, 46, 395-407.

Fisher, C. B., Wallace, S. A., \& Fenton, R. E. (2000). Discrimination distress during adolescence. Journal of Youth and Adolescence, 29, 679-695.

Hughes, D. (2003). Correlates of African American and Latino parents' messages to children about ethnicity and race: A comparative study of racial socialization. American Journal of Community Psychology, 31, 15-33.

Hughes, D., \& Chen, L. (1997). When and what parents tell children about race: An examination of race-related socialization among African American families. Applied Developmental Science, 1, 200-214.

Hughes, D., \& Chen, L. (1999). The nature of parents' race related communications to children: A developmental perspective. In L. Balter \& C. Tamis-LeMonda (Eds.), Child psychology: A handbook of contemporary issues (pp. 467-490). Philadelphia, PA: Taylor \& Francis.

Hughes, D., \& Johnson, D. (2001). Correlates in children's experiences of parents' racial socialization behaviors. Journal of Marriage and Family, 63, 981-995.

Nyborg, V., \& Curry, J. (2003). The impact of perceived racism: Psychological symptoms among African American boys. Journal of Clinical Child and Adolescent Psychology, 32, 258-266.

Prelow, H., Danoff-Burg, S., Swenson, R., \& Pulgiano, D. (2004). The impact of ecological risk and perceived discrimination on the psychological adjustment of African American and European American youth. Journal of Community Psychology, 32, 375-389.

Rosenberg, M. (1965). Society and the adolescent self-image. Princeton, NJ: Princeton University Press. 
Rosenbloom, S. R., \& Way, N. (2004). Experiences of discrimination among African American, Asian American, and Latino adolescents in an urban high school. Youth and Society, 35, 420-451.

Sellers, R. M., \& Shelton, J. N. (2003). The role of racial identity in perceived discrimination. Journal of Personality and Social Psychology, 84, 1079-1092.

Spencer, M. B. (1999). Social and cultural influences on school adjustment: The application of an identity-focused cultural ecological perspective. Educational Psychologist, 34, 43-57.

Stevenson, H., Reed, J., Bodison, P., \& Bishop, A. (1997). Racism stress management: Racial socialization beliefs and the experience of depression and anger in African American youth. Youth and Society, 29, 172-222.

Wong, C., Eccles, J., \& Sameroff, A. (2003). The influence of ethnic discrimination and ethnic identification on African American adolescents' school and socioemotional adjustment. Journal of Personality, 71, 1197-1232. 